\title{
The Uses of Acetylacetone to Synthesize Pyridin-2-
} One Derivatives

\author{
L. Rateb, M.M. Mishrikey, G.A. Mina*, B.A. Silwanis and \\ N.Y. Megally Abdo** \\ Chemistry Department, Faculty of Science, and "Chemistry \\ Department, Faculty of Education, Alexandria University, \\ Alexandria 21526, Egypt.
}

\begin{abstract}
SERIES of pyridin-2-one derivatives have been prepared from the reaction of acetyl acetone and cyanoacetohydrazide or dialkyl carbocyanohydrazodithioates as well as $2 \mathrm{H}$-pyran-ones. Some reactions of the pyridin-2-one were also studied. The synthesized compounds were structurally characterized on the basis of UV, IR, ${ }^{1} \mathrm{H}$ $\mathrm{NMR},{ }^{13} \mathrm{C}$ NMR, mass spectrometry and elemental analyses.
\end{abstract}

Keywords: Pyridin-2-one, Cyanoacetohydrazide, Dialkyl carbocyanohydrazodithioates and $2 \mathrm{H}$-pyran-ones.

The synthesis of pyridin-2-one derivatives is a continuing area of interest due to the number of biologically active molecules containing this moiety ${ }^{(1-5)}$. Natural compounds with this structure have emerged during the last ten years as potent antitumor, antibacterial activity ${ }^{(6,7)}$, antifungal ${ }^{(8)}$, antiinflammatory, analgesic, antipyfuztic $^{(9)}$, antiviral ${ }^{(10)}$, psychotherapeutic ${ }^{(11)}$ and evaluated as human rhinovirus (HRV) 3C-protease $(3 \mathrm{CP})$ inhibitors ${ }^{(12)}$. Moreover, pyridones are the key intermediates in the synthesis of the corresponding pyridines ${ }^{(13)}$, they have been prepared by numerous methods ${ }^{(14)}$. Many literature sources describe more general approaches involving the condensation of unsaturated ketones with methylene active amides, using cyanoacetamide ${ }^{(15-19)}$ or by the reaction of 2 pyrones with ammonia, amines as well as hydrazines ${ }^{(20)}$.

Despite this large number of existing methods for the synthesis of 2 pyridones, we report here the synthesis of 4,6-disubstituted-2-pyridone derivatives and characterization of their structures by different spectral tools.

\section{Chemistry}

\section{Experimental}

All melting points were determined on a Stuart apparatus and the values given are uncorrected. IR spectra $\left(\mathrm{KBr}, \mathrm{cm}^{-1}\right)$ were determined on a PerkinElmer 1430 spectrophotometer (Microanalysis Center, Alexandria University, Egypt). Electronic spectra were measured with a Perkin-Elmer Lambada 4B spectrophotometer (Microanalysis Center, Alexandria University, Egypt). ${ }^{1} \mathrm{H}$ NMR and ${ }^{13} \mathrm{C}$ NMR spectra were recorded on Varian EM-390 at $90 \mathrm{MHz}$ and Varian Gemini $300 \mathrm{MHz}$, operating at $75.50 \mathrm{MHz}$, respectively (Microanalysis Center, Alexandria University, Egypt)) using TMS as internal standard.

\footnotetext{
${ }^{\#}$ Corresponding author: E-mail: nadiamegally@yahoo.com
} 
Chemical shift values are recorded in ppm ondscale. Mass spectra were recorded at $70 \mathrm{eV}$ with an AEL, MS-9 spectrometer (Microanalysis Center, Cairo University, Egypt). Elemental analyses were carried out at the Microanalysis Center, Cairo University, Egypt; found values were within $\pm 0.35 \%$ of the theoretical ones. Progress of the reactions was monitored using thin layer chromatography (TLC) sheets precoated with UV fluorescent silica gel Merck 60F 254 and was visualized using UV lamp.

1-Amino-4,6-dimethyl-2-oxo-1,2-dihydropyridine-3-carbonitrile (2)

A solution of 2-isocyanoacetohydrazide (1) $(11.5 \mathrm{~g}, 0.116 \mathrm{~mol})$ in ethanol $(100 \mathrm{ml})$ was gradually added to a solution of acetylacetone $(11.6 \mathrm{~g}, 0.116 \mathrm{~mol})$ in ethanol $(50 \mathrm{ml})$ containing one drop of piperidine. After refluxing the reaction mixture for $10 \mathrm{~min}$, and cooling, the separated product was filtered off, washed with ethanol and crystallized from ethanol.

Yield: $87 \%$; m.p.: $172-174{ }^{\circ} \mathrm{C}$; UV $\left(\lambda_{\max } \mathrm{nm}\right): \mathrm{MeOH}, 395,338,224$; $(0.1$ M) $\mathrm{MeOH} \backslash \mathrm{H}_{2} \mathrm{SO}_{4}, 395,338,224$; (0.1 M) $\mathrm{MeOH} \backslash \mathrm{NaOCH}_{3}, 395,338,224,206$; IR $\left(\mathrm{KBr}, \mathrm{cm}^{-1}\right): 3283,3201\left(\mathrm{NH}_{2}\right), 2218(\mathrm{CN}), 1621(\mathrm{C}=\mathrm{O}) ;{ }^{1} \mathrm{H}$ NMR (DMSO$\left.d_{6}\right): \delta 2.35\left(\mathrm{~s}, 3 \mathrm{H}, \mathrm{CH}_{3}\right), 2.45\left(\mathrm{~s}, 3 \mathrm{H}, \mathrm{CH}_{3}\right), 6.05\left(\mathrm{~s}, 2 \mathrm{H}, \mathrm{NH}_{2}, \mathrm{D}_{2} \mathrm{O}\right.$ exchangeable), 6.25 (s, 1H, pyridine $\mathrm{H}-5)$; MS: $m / z$ (\%) $163\left(\mathrm{M}^{+}, 49.9\right)$. Anal. Calcd. for $\mathrm{C}_{8} \mathrm{H}_{9} \mathrm{~N}_{3} \mathrm{O}$ : C, 58.88; H, 5.56; N, 25.75. Found: C, 58.62; H, 5.70; N, 26.01.

4,6-Dimethyl-2-oxo-1,2-dihydropyridine-3-carbonitrile (3)

A solution of 1-amino-4,6-dimethyl-2-oxo-1,2-dihydropyridine-3-carbonitrile (2) $(2 \mathrm{~g}, 0.014 \mathrm{~mol})$ in glacial acetic acid $(8 \mathrm{ml})$ was treated dropwise with an aqueous solution of sodium nitrite $(0.5 \mathrm{gm})$. The separated product was filtered off, washed with ethanol and crystallized from acetic acid.

Yield: $75 \%$; m.p.: $284-286{ }^{\circ} \mathrm{C}$; UV $\left(\lambda_{\max } \mathrm{nm}\right): \mathrm{MeOH}, 395,327,237,213$; $\mathrm{MeOH} \backslash \mathrm{H}_{2} \mathrm{SO}_{4}, 395,327,238,213 ; \mathrm{MeOH} \backslash N a O C H_{3}, 395,326,246,214 ;$; IR $\left(\mathrm{KBr}, \mathrm{cm}^{-1}\right): 3287(\mathrm{NH}), 2305(\mathrm{CN}), 1660(\mathrm{C}=\mathrm{O}) ;{ }^{1} \mathrm{H}$ NMR (DMSO- $\left.d_{6}\right): \delta 2.24$ $\left(\mathrm{s}, 3 \mathrm{H}, \mathrm{CH}_{3}\right), 2.35$ (s, 3H, $\left.\mathrm{CH}_{3}\right), 6.05(\mathrm{~s}, 1 \mathrm{H}$, pyridine $\mathrm{C}-5), 12.14$ (s, $1 \mathrm{H}, \mathrm{NH}$, $\mathrm{D}_{2} \mathrm{O}$ exchangeable); $\mathrm{MS}: \mathrm{m} / z(\%) 148\left(\mathrm{M}^{+}, 55.9\right)$. Anal. Calcd. for $\mathrm{C}_{8} \mathrm{H}_{8} \mathrm{~N}_{2} \mathrm{O}: \mathrm{C}$, 64.85; H, 5.44; N, 18.91. Found: C, 64.63; H, 5.58; N, 18.78.

4,6-Dimethyl-5-nitro-2-oxo-1,2-dihydropyridine-3-carbonitrile (4)

A mixture of nitric $(\mathrm{d} 1.41 ; 3 \mathrm{ml})$ and sulfuric $(\mathrm{d} 1.84 ; 3 \mathrm{ml})$ acids in glacial acetic acid $(10 \mathrm{ml})$ was gradually added to a solution of 1-amino-4,6-dimethyl-2oxo-1,2-dihydropyridine-3-carbonitrile (2) (2 g, $0.014 \mathrm{~mol})$ in glacial acetic acid $(15 \mathrm{ml})$. The reaction mixture was warmed at $50^{\circ} \mathrm{C}$ for $15 \mathrm{~min}$, then poured into cold water with stirring. The separated product was filtered washed with cold water, dried and crystallized from acetic acid.

Yield: $77 \%$; m.p.: $258-260{ }^{\circ} \mathrm{C}$; UV ( $\left.\lambda_{\max } \mathrm{nm}\right): \mathrm{MeOH}, 390$ (sh), 326, 232 (sh), 212; $\mathrm{MeOH} \backslash \mathrm{H}_{2} \mathrm{SO}_{4}, 390$ (sh), 327, 233, 209; $\mathrm{MeOH} \backslash \mathrm{NaOCH}_{3}, 370$ (sh), 326, 239, 207; IR (KBr, cm$\left.{ }^{-1}\right): 3288(\mathrm{NH}), 2228(\mathrm{CN}), 1654(\mathrm{C}=\mathrm{O}) ;{ }^{1} \mathrm{H}$ NMR

Egypt. J. Chem. 58, No. 6 (2015) 
(DMSO-d $\left.d_{6}\right): \delta 2.32\left(\mathrm{~s}, 6 \mathrm{H}, 2 \mathrm{CH}_{3}\right), 10.22\left(\mathrm{~s}, 1 \mathrm{H}, \mathrm{NH}, \mathrm{D}_{2} \mathrm{O}\right.$ exchangeable); MS: $\mathrm{m} / \mathrm{z}(\%) 193\left(\mathrm{M}^{+}, 24.1\right)$. Anal. Calcd. for $\mathrm{C}_{8} \mathrm{H}_{7} \mathrm{~N}_{3} \mathrm{O}_{3}$ : C, 49.74; $\mathrm{H}, 3.65 ; \mathrm{N}, 21.75$. Found: C, 49.52; H, 3.81; N, 22.01.

1-(4-Hydroxypent-3-en-2-ylidene)amino)-4,6-dimethyl-2-oxo-1,2 dihydropyridine -3carbonitrile (5)

A solution of 1-amino-4,6-dimethyl-2-oxo-1,2-dihydropyridine-3-carbonitrile (2) $(1 \mathrm{~g}, 0.007 \mathrm{~mol})$ in ethanol $(10 \mathrm{ml})$ was added to a solution of acetylacetone $(1.2 \mathrm{~g}, 0.012 \mathrm{~mol})$ in ethanol $(10 \mathrm{ml})$ acidified with acetic acid and the reaction mixture was refluxed for $10 \mathrm{hr}$. On cooling, the solid product was separated, filtered and crystallized from ethanol.

Yield: $79 \%$; m.p.: $146-148{ }^{\circ} \mathrm{C}$; UV $\left(\lambda_{\max } \mathrm{nm}\right): \mathrm{MeOH}, 340,284,216$; $\mathrm{MeOH} \backslash \mathrm{H}_{2} \mathrm{SO}_{4}, 339,270,225 ; \mathrm{MeOH} \backslash N a O C H_{3}, 341$ (sh), 302, 226 (sh), 205; IR $\left(\mathrm{KBr}, \mathrm{cm}^{-1}\right): 3100\left(\mathrm{CH}\right.$ aromatic), $2214(\mathrm{CN}), 1664(\mathrm{C}=\mathrm{O})$; ${ }^{1} \mathrm{H}$ NMR (DMSO$\left.d_{6}\right): \delta 1.61\left(\mathrm{~s}, 3 \mathrm{H}, \mathrm{CH}_{3}\right), 1.74\left(\mathrm{~s}, 3 \mathrm{H}, \mathrm{CH}_{3}\right), 2.44\left(\mathrm{~s}, 3 \mathrm{H}, \mathrm{CH}_{3}\right), 2.62\left(\mathrm{~s}, 3 \mathrm{H}, \mathrm{CH}_{3}\right)$, $5.35(\mathrm{~s}, 1 \mathrm{H}, \mathrm{CH}), 6.35(\mathrm{~s}, 1 \mathrm{H}$, pyridine $\mathrm{H}-5), 11.54\left(\mathrm{~s}, 1 \mathrm{H}, \mathrm{OH}, \mathrm{D}_{2} \mathrm{O}\right.$ exchangeable); MS: $m / z$ (\%) $245\left(\mathrm{M}^{+}, 34.8\right)$. Anal. Calcd. for $\mathrm{C}_{13} \mathrm{H}_{15} \mathrm{~N}_{3} \mathrm{O}_{2}: \mathrm{C}$, 63.66; H, 6.16; N, 17.13. Found: C, 63.84; H, 6.32; N, 17.40.

General procedure for the synthesis of $6 a, b$

A solution of $\mathrm{KOH}(5.6 \mathrm{~g}, 0.2 \mathrm{~mol})$ in methanol $(100 \mathrm{ml})$ was gradually $(10$ min) added to a suspension of cyanoacetohydrazide (1) $(9.9 \mathrm{~g}, 0.1 \mathrm{~mol})$ in methanol $(100 \mathrm{ml})$ with stirring at $0{ }^{\circ} \mathrm{C}$, whereby the hydrazide completely dissolved. The reaction mixture was then treated dropwise $(10 \mathrm{~min})$ with $\mathrm{CS}_{2}$ $(7.6 \mathrm{~g}, 0.1 \mathrm{~mol})$ at $0{ }^{\circ} \mathrm{C}$. After complete addition of $\mathrm{CS}_{2}$, the separated solid of potassium 2-(2-isocyanoacetyl)hydrazinecarbodithioate was filtered, washed with cold ethanol and dried (19 g, Yield: $84 \%)$.

A suspension of the crude potassium 2-(2-isocyanoacetyl) hydrazinecarbodithioate $(21 \mathrm{~g}, 0.09 \mathrm{~mol})$ in methanol $(100 \mathrm{ml})$ was gradually treated with stirring at $0{ }^{\circ} \mathrm{C}$ with an ethanolic solution of either methyl iodide $(21.9 \mathrm{~g}, 0.186 \mathrm{~mol})$ or ethyl iodide $(24 \mathrm{~g}, 0.186 \mathrm{~mol})$ in the course of $20 \mathrm{~min}$. The reaction mixture was then kept overnight in the ice-chest with occasional shaking. The separated product was filtered and crystallized from methanol.

Dimethyl (2-isocyanoacetyl)carbonohydrazonodithioate (6a)

Yield: $79 \%$; m.p.: $127-129{ }^{\circ} \mathrm{C}$; UV $\left(\lambda_{\max } \mathrm{nm}\right)$ : MeOH, 271, 227, 215 (sh);

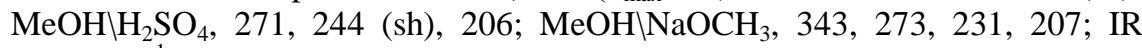
$\left(\mathrm{KBr}, \mathrm{cm}^{-1}\right): 3169(\mathrm{NH}), 3013(\mathrm{CH}$ aromatic), $2255(\mathrm{CN}), 1646(\mathrm{C}=\mathrm{O}), 1572$ $(\mathrm{C}=\mathrm{N}) ;{ }^{1} \mathrm{H}$ NMR (DMSO- $\left.d_{6}\right): \delta 2.43\left(\mathrm{~s}, 3 \mathrm{H}, \mathrm{CH}_{3}\right), 2.51\left(\mathrm{~s}, 3 \mathrm{H}, \mathrm{CH}_{3}\right), 3.85(\mathrm{dd}$, $\left.2 \mathrm{H}, J=27.7 \mathrm{~Hz}, \mathrm{CH}_{2}\right), 10.55$ (s, 1H, NH, D $\mathrm{O}$ exchangeable); MS: $m / z$ (\%) 203 $\left(\mathrm{M}^{+}\right.$, 75.4). Anal. Calcd. for $\mathrm{C}_{6} \mathrm{H}_{9} \mathrm{~N}_{3} \mathrm{OS}_{2}$ : C, 35.45; H, 4.46; N, 20.67; S, 31.55. Found: C, 35.63; H, 4.71; N, 20.51; S, 31.33. 
Diethyl (2-isocyanoacetyl)carbonohydrazonodithioate (6b)

Yield: $84 \%$; m.p.: $73-75{ }^{\circ} \mathrm{C}$; UV $\left(\lambda_{\max } \mathrm{nm}\right): \mathrm{MeOH}, 275,227,212$;

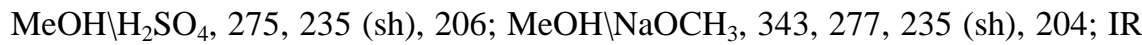
$\left(\mathrm{KBr}, \mathrm{cm}^{-1}\right): 3173(\mathrm{NH}), 3089(\mathrm{CH}$ aromatic), $2255(\mathrm{CN}), 1682(\mathrm{C}=\mathrm{O}), 1646$ $(\mathrm{C}=\mathrm{N}) ;{ }^{1} \mathrm{H}$ NMR (DMSO- $\left.d_{6}\right): \delta 1.35\left(\mathrm{t}, 6 \mathrm{H}, J=7.0 \mathrm{~Hz}, 2 \mathrm{CH}_{2}-\mathrm{CH}_{3}\right), 3.15(\mathrm{q}, 4 \mathrm{H}$, $\left.J=7.2 \mathrm{~Hz}, 2 \mathrm{CH}_{2}-\mathrm{CH}_{3}\right), 3.91$ (dd, $\left.2 \mathrm{H}, J=22.2 \mathrm{~Hz}, \mathrm{CH}_{2}\right), 10.55$ (br.s, $1 \mathrm{H}, \mathrm{NH}$, $\mathrm{D}_{2} \mathrm{O}$ exchangeable) ${ }^{13} \mathrm{C}$ NMR (DMSO- $d_{6}$ ): $\delta 13.8,15.1,24.4,26.1,26.7,116.1$, 158.2, 165.4; MS: $\mathrm{m} / \mathrm{z}(\%) 232\left(\mathrm{M}^{+1}, 100\right)$. Anal. Calcd. for $\mathrm{C}_{8} \mathrm{H}_{13} \mathrm{~N}_{3} \mathrm{OS}_{2}: \mathrm{C}$, 41.53; H, 5.66; N, 18.16; S, 27.72. Found: C, 41.32; H, 5.70; N, 18.27; S, 27.54.

General procedure for the synthesis of $7 a, b$

A solution of dimethyl (2-isocyanoacetyl)carbonohydrazonodithioate (6a) $(1 \mathrm{~g}, 0.005 \mathrm{~mol})$ in ethanol $(10 \mathrm{ml})$ was refluxed for $30 \mathrm{~min}$ with an ethanolic solution of either benzaldhyde $(0.5 \mathrm{ml}, 0.005 \mathrm{~mol})$ or benzil $(1 \mathrm{~g}, 0.005 \mathrm{~mol})$ containing one drop of piperidine. On cooling, the solid product was separated, filtered, washed with cold ethanol and crystallized from ethanol.

Dimethyl (2-cyano-3-phenylacryloyl)carbonohydrazonodithioate (7a)

Yield: $91 \%$; m.p.: $135-137{ }^{\circ} \mathrm{C}$; UV ( $\left.\lambda_{\max } \mathrm{nm}\right): \mathrm{MeOH}, 307,222$ (sh), 205;

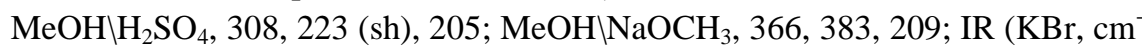
$\left.{ }^{1}\right)$ : $3307(\mathrm{NH}), 3000\left(\mathrm{CH}\right.$ aromatic), $2204(\mathrm{CN}), 1687(\mathrm{C}=\mathrm{O}), \mathrm{C}=\mathrm{N}(1646) ;{ }^{1} \mathrm{H}$ NMR (DMSO- $\left.d_{6}\right): \delta 2.52\left(\mathrm{~s}, 3 \mathrm{H}, \mathrm{CH}_{3}\right), 2.61\left(\mathrm{~s}, 3 \mathrm{H}, \mathrm{CH}_{3}\right), 7.43-7.98(\mathrm{~m}, 5 \mathrm{H}$, $\mathrm{Ar}-\mathrm{H}), 8.15$ (s, $1 \mathrm{H}, \underline{\mathrm{HC}}=\mathrm{C}-$-) $10.62\left(\mathrm{~s}, 1 \mathrm{H}, \mathrm{NH}, \mathrm{D}_{2} \mathrm{O}\right.$ exchangeable); MS: $\mathrm{m} / \mathrm{z}$ (\%) $291\left(\mathrm{M}^{+}\right.$, 14.9). Anal. Calcd. for $\mathrm{C}_{13} \mathrm{H}_{13} \mathrm{~N}_{3} \mathrm{OS}_{2}$ : C, 53.58; H, 4.50; N, 14.42; S, 22.01. Found: C, 53.74; H, 4.71; N, 14.72; S, 21.91 (7b)

$N^{\prime}$-(Bis(methylthio)methylene)-2-cyano-4-oxo-3,4-diphenylbut-2-enehydrazonic acid

Yield: $92 \%$; m.p.: $198-200{ }^{\circ} \mathrm{C}$; UV $\left(\lambda_{\max } \mathrm{nm}\right)$ : $\mathrm{MeOH}, 580,387,345,292$;

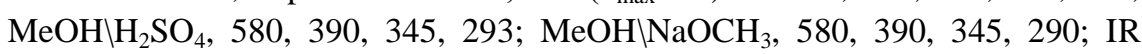
$\left(\mathrm{KBr}, \mathrm{cm}^{-1}\right)$ : $3301(\mathrm{NH}), 3076(\mathrm{CH}$ aromatic), $2219(\mathrm{CN}), 1680,1889(2 \mathrm{C}=\mathrm{O})$, $\mathrm{C}=\mathrm{N}(1592) ;{ }^{1} \mathrm{H}$ NMR $\left(\mathrm{DMSO}-d_{6}\right): \delta 2.11\left(\mathrm{~s}, 3 \mathrm{H}, \mathrm{CH}_{3}\right), 2.42\left(\mathrm{~s}, 3 \mathrm{H}, \mathrm{CH}_{3}\right), 7.21-$ $7.64(\mathrm{~m}, 10 \mathrm{H}, \mathrm{Ar}-\mathrm{H}), 7.90$ (s, $1 \mathrm{H}, \mathrm{OH}, \mathrm{D}_{2} \mathrm{O}$ exchangeable); ${ }^{13} \mathrm{C}$ NMR (DMSO$\left.d_{6}\right): \delta 19.8,20.1,98.6,110.3,119.3,131.4,132.9,133.1,135.0,136.1,137.6$, 138.2, 142.9, 162.9, 173.1, 179.1; MS: $m / z$ (\%) $395\left(\mathrm{M}^{+}\right.$, 54.3). Anal. Calcd. for $\mathrm{C}_{20} \mathrm{H}_{17} \mathrm{~N}_{3} \mathrm{O}_{2} \mathrm{~S}_{2}$ : C, 60.74; H, 4.33; N, 10.62; S, 16.22. Found: C, 60.47; H, 4.60; N, 10.72; S, 15.94.

General procedure for the synthesis of $8 a, b$

when a solution of either $6 \mathrm{a}(2 \mathrm{~g}, 0.01 \mathrm{~mol})$ or $6 \mathrm{~b}(5 \mathrm{~g}, 0.02 \mathrm{~mol})$ in methanol $(20 \mathrm{ml})$ was treated with a methanolic solution of acetylacetone $(0.79 \mathrm{~g}, 0.01$

Egypt. J. Chem. 58, No. 6 (2015) 
mol or $2.5 \mathrm{~g}, 0.02 \mathrm{~mol}$ ) and two drops of piperidine. The reaction mixture was heated under reflux for $30 \mathrm{~min}$. On cooling the solid product was separated, filtered, washed with cold methanol and crystallized from methanol.

Dimethyl (3-cyano-4,6-dimethyl-2-oxopyridin-1(2H)-yl) carbonimidodithioate (8a)

Yield: $84 \%$; m.p.: $184-186{ }^{\circ} \mathrm{C}$; UV $\left(\lambda_{\max } \mathrm{nm}\right)$ : $\mathrm{MeOH}, 341,225$; $\mathrm{MeOH} \backslash \mathrm{H}_{2} \mathrm{SO}_{4}, 341,235 ; \mathrm{MeOH} \backslash N a O C H_{3}, 341,225,206 ; \mathrm{IR}\left(\mathrm{KBr}, \mathrm{cm}^{-1}\right): 2953$ (CH aromatic), $2215(\mathrm{CN}), 1645(\mathrm{C}=\mathrm{O}), 1576(\mathrm{C}=\mathrm{N}) ;{ }^{1} \mathrm{H}$ NMR (DMSO- $\left.d_{6}\right): \delta$ $2.20\left(\mathrm{~s}, 3 \mathrm{H}, \mathrm{CH}_{3}\right), 2.35$ (s, $\left.3 \mathrm{H}, \mathrm{CH}_{3}\right), 2.55$ (s, $\left.3 \mathrm{H}, \mathrm{SCH}_{3}\right), 2.65$ (s, 3H, $\mathrm{SCH}_{3}$ ), 6.32 (s, $1 \mathrm{H}$, pyridine $\mathrm{H}-5) ;{ }^{13} \mathrm{C}$ NMR (DMSO- $d_{6}$ ): $\delta 13.9,15.1,18.5,20.4,99.7$, 108.4, 116.4, 150.4, 155.4, 157.1, 180.1; MS: $m / z$ (\%) 268 ( $\mathrm{M}^{+1}$, 100). Anal. Calcd. for $\mathrm{C}_{11} \mathrm{H}_{13} \mathrm{~N}_{3} \mathrm{OS}_{2}$ : C, 49.41; H, 4.90; N, 15.72; S, 23.99. Found: C, 49.61; $\mathrm{H}, 5.12 ; \mathrm{N}, 15.52 ; \mathrm{S}, 23.78$.

Diethyl (3-cyano-4,6-dimethyl-2-oxopyridin-1(2H)-yl) carbonimidodithioate (8b)

Yield: $78 \%$; m.p.: $98-100{ }^{\circ} \mathrm{C}$; UV $\left(\lambda_{\max } \mathrm{nm}\right)$ : $\mathrm{MeOH}, 342,223$; $\mathrm{MeOH} \backslash \mathrm{H}_{2} \mathrm{SO}_{4}, 342,237 ; \mathrm{MeOH} \backslash N a O C H_{3}, 342,238,206$; IR $\left(\mathrm{KBr}, \mathrm{cm}^{-1}\right): 2972$ (CH aromatic), $2215(\mathrm{CN}), 1659(\mathrm{C}=\mathrm{O}), 1587(\mathrm{C}=\mathrm{N}) ;{ }^{1} \mathrm{H}$ NMR (DMSO- $\left.d_{6}\right): \delta$ $1.35\left(\mathrm{t}, 6 \mathrm{H}, J=7.0 \mathrm{~Hz}, 2 \mathrm{CH}_{2}-\mathrm{CH}_{3}\right), 2.20\left(\mathrm{~s}, 3 \mathrm{H}, \mathrm{CH}_{3}\right), 2.35$ (s, $\left.3 \mathrm{H}, \mathrm{CH}_{3}\right), 3.15$ (q, $\left.4 \mathrm{H}, J=7.2 \mathrm{~Hz}, 2 \mathrm{CH}_{2}-\mathrm{CH}_{3}\right), 6.35$ (s, $1 \mathrm{H}$, pyridine $\mathrm{H}-5$ ); MS: $\mathrm{m} / z$ (\%) $295\left(\mathrm{M}^{+}\right.$, 55.3). Anal. Calcd. for $\mathrm{C}_{13} \mathrm{H}_{17} \mathrm{~N}_{3} \mathrm{OS}_{2}$ : C, 52.85; H, 5.80; N, 14.22; S, 21.71. Found: C, 52.62; H, 6.01; N, 14.50; S, 21.44 .

Dimethyl (5-bromo-3-cyano-4,6-dimethyl-2-oxopyridin-1(2H)-yl) carbonimidodithioate (9)

A solution of bromine $(3.2 \mathrm{~g}, 0.02 \mathrm{~mol})$ in carbon tetrachloride $(20 \mathrm{ml})$ was gradually added to a suspension of compound $7 \mathrm{a}(2.7 \mathrm{~g}, 0.01 \mathrm{~mol})$ in $\mathrm{CCl}_{4}$ $(20 \mathrm{ml})$ with stirring for $1 \mathrm{hr}$ at room temperature. The carbon tetrachloride solution was washed with water, dried over anhydrous sodium sulfate and evaporated under reduced pressure. The residual oily product solidified on treatment with ethanol and crystallized from ethanol.

Yield: $86 \%$; m.p.: $122-125{ }^{\circ} \mathrm{C}$; UV $\left(\lambda_{\max } \mathrm{nm}\right): \mathrm{MeOH}, 352,230$; $\mathrm{MeOH} \backslash \mathrm{H}_{2} \mathrm{SO}_{4}, 352,232 ; \mathrm{MeOH} \mathrm{NaOCH}_{3}, 352,233 ; \mathrm{IR}\left(\mathrm{KBr}, \mathrm{cm}^{-1}\right): 2929(\mathrm{CH}$ aromatic), $2220(\mathrm{CN}), 1665(\mathrm{C}=\mathrm{O}), 1588(\mathrm{C}=\mathrm{N}) ;{ }^{1} \mathrm{H}$ NMR (DMSO- $\left.d_{6}\right): \delta 2.42$ (s, $3 \mathrm{H}, \mathrm{CH}_{3}$ ), 2.45 (s, $3 \mathrm{H}, \mathrm{CH}_{3}$ ), 2.53 (s, $\left.3 \mathrm{H}, \mathrm{SCH}_{3}\right), 2.61$ (s, $\left.3 \mathrm{H}, \mathrm{SCH}_{3}\right) ; \mathrm{MS}: \mathrm{m} / z$ (\%) $346\left(\mathrm{M}^{+}\right.$, 12.7). Anal. Calcd. for $\mathrm{C}_{11} \mathrm{H}_{12} \mathrm{BrN}_{3} \mathrm{OS}_{2}: \mathrm{C}, 38.15 ; \mathrm{H}, 3.49 ; \mathrm{Br}$, 23.08; N, 12.14; S, 18.52. Found: C, 38.41; H, 3.72; Br, 23.11; N, 12.01; S, 18.43 
3-Bromo-4,6-diphenyl-2H-pyran-2-one (11a)

A suspension of 4,6-diphenyl-2H-pyran-2-one (10) $(1.24 \mathrm{~g}, 0.005 \mathrm{~mol})$ in chloroform $(25 \mathrm{ml})$ was treated with a solution of bromine $(0.265 \mathrm{~g}, 0.005 \mathrm{~mol})$ in chloroform $(8 \mathrm{ml})$ and the reaction mixture warmed for $15 \mathrm{~min}$. The chloroform solution was then washed with water, dried over anhydrous sodium sulfate and evaporated under reduced pressure. The residual product was crystallized from benzene-n-hexane.

Yield: $85 \%$; m.p.: $128-130{ }^{\circ} \mathrm{C}$; UV $\left(\lambda_{\max } \mathrm{nm}\right): \mathrm{MeOH}, 349,254,205$; $\mathrm{MeOH} \backslash \mathrm{H}_{2} \mathrm{SO}_{4}, 349,254,205 ; \mathrm{MeOH} \backslash N a O C H_{3}, 349,254,205$; IR $\left(\mathrm{KBr}, \mathrm{cm}^{-1}\right)$ : 2945 (CH aromatic), $1723(\mathrm{C}=\mathrm{O}) ;{ }^{1} \mathrm{H}$ NMR (DMSO-d $)$ ): $\delta 6.71(\mathrm{~s}, 1 \mathrm{H}$, pyran H5), 7.41-7.87 (m, 10H, Ar-H); MS: m/z (\%) $327\left(\mathrm{M}^{+}, 10.2\right)$. Anal. Calcd. for $\mathrm{C}_{17} \mathrm{H}_{11} \mathrm{BrO}_{2}$ : C, 62.41; H, 3.39; Br, 24.42. Found: C, 62.23; H, 3.70; Br, 24.12.

\section{3-Iodo-4,6-diphenyl-2H-pyran-2-one (11b)}

A solution of iodine monochloride $(0.4 \mathrm{~g}, 0.002 \mathrm{~mol})$ in dry chloroform $(10 \mathrm{ml})$ was gradually added to a solution of 4,6-diphenyl-2H-pyran-2-one $(0.5$ $\mathrm{g}, 0.002 \mathrm{~mol})$ in dry chloroform $(5 \mathrm{ml})$ with stirring for $30 \mathrm{~min}$ at room temperature. The precipitated product was filtered and crystallized from ethanol.

Yield: $67 \%$; m.p.: $258-260{ }^{\circ} \mathrm{C}$; IR $\left(\mathrm{KBr}, \mathrm{cm}^{-1}\right): 2986(\mathrm{CH}$ aromatic), 1720 $(\mathrm{C}=\mathrm{O}) ;{ }^{1} \mathrm{H}$ NMR $\left(\mathrm{DMSO}-d_{6}\right): \delta 6.82(\mathrm{~s}, 1 \mathrm{H}$, pyran H-5), 7.43-7.91 $(\mathrm{m}, 10 \mathrm{H}, \mathrm{Ar}-$ H); MS: $m / z(\%) 374\left(\mathrm{M}^{+}, 100\right)$. Anal. Calcd. for $\mathrm{C}_{17} \mathrm{H}_{11} \mathrm{IO}_{2}$ : C, 54.57; H, 2.96 . Found: C, 54.35; H, 2.81 .

\section{3-Nitro-4,6-diphenyl-2H-pyran-2-one (11c)}

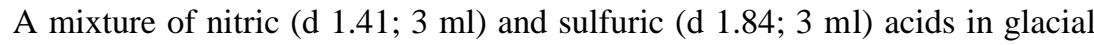
acetic acid $(10 \mathrm{ml})$ was gradually added to a solution of 4,6-diphenyl-2H-pyran2-one (10) (2 g, $0.08 \mathrm{~mol})$ in glacial acetic acid $(15 \mathrm{ml})$. The reaction mixture was warmed at $50^{\circ} \mathrm{C}$ for $15 \mathrm{~min}$, cooled, poured into cold water with stirring. The separated product was filtered, washed with cold water, dried and crystallized from acetic acid.

Yield: $83 \%$; m.p.: $185-187{ }^{\circ} \mathrm{C}$; IR $\left(\mathrm{KBr}, \mathrm{cm}^{-1}\right): 2959(\mathrm{CH}$ aromatic), 1714 $(\mathrm{C}=\mathrm{O}), 1534,1340(\mathrm{NO} 2) ;{ }^{1} \mathrm{H}$ NMR (DMSO- $\left.d_{6}\right): \delta 6.85(\mathrm{~s}, 1 \mathrm{H}$, pyran H-5), 7.41-7.98 (m, 10H, Ar-H); MS: m/z (\%) $293\left(\mathrm{M}^{+}, 100\right)$. Anal. Calcd. for $\mathrm{C}_{17} \mathrm{H}_{11} \mathrm{NO}_{4}$ : C, 69.62; H, 3.78; N, 4.78. Found: C, 69.69; H, 4.01; N, 4.99.

\section{1-Amino-4,6-diphenylpyridin-2(1H)-one (12)}

A solution of 4,6-diphenyl-2H-pyran-2-one (10) $(0.5 \mathrm{~g}, 0.002 \mathrm{~mol})$ in ethanol $(10 \mathrm{ml})$ was refluxed with $85 \%$ hydrazine hydrate $(2 \mathrm{ml})$ for $1.5 \mathrm{hr}$. The reaction

Egypt. J. Chem. 58, No. 6 (2015) 
mixture was then diluted with water, the separated product was filtered, washed with cold water, dried and crystallized from benzene-light petroleum (b.p. 60-80).

Yield: $58 \%$; m.p.: $163-165^{\circ} \mathrm{C}$; IR $\left(\mathrm{KBr}, \mathrm{cm}^{-1}\right): 3268,3195\left(\mathrm{NH}_{2}\right), 2945(\mathrm{CH}$ aromatic), $1645(\mathrm{C}=\mathrm{O}) ;{ }^{1} \mathrm{H}$ NMR $\left(\mathrm{DMSO}-d_{6}\right): \delta 5.07$ (br s, $2 \mathrm{H}, \mathrm{NH}_{2}, \mathrm{D}_{2} \mathrm{O}$ exchangeable), $6.45(\mathrm{~d}, 1 \mathrm{H}, J=2.6 \mathrm{~Hz}$, pyridine $\mathrm{H}-5), 6.86(\mathrm{~d}, 1 \mathrm{H}, J=2.6 \mathrm{~Hz}$, pyridine H-3), 7.23-7.74 (m, 10H, Ar-H),; MS: m/z (\%) $262\left(\mathrm{M}^{+}, 54.6\right)$. Anal. Calcd. for $\mathrm{C}_{17} \mathrm{H}_{14} \mathrm{~N}_{2} \mathrm{O}$ : C, 77.84; H, 5.38; N, 10.68. Found: C, 77.63; H, 5.17; N, 10.89 .

4,6-Diphenylpyridin-2(1H)-one (13)

A solution of 1-amino-4,6-diphenylpyridin-2(1H)-one (12) (0.2 g, 0.0008 $\mathrm{mol})$ in glacial acetic acid $(8 \mathrm{ml})$ was treated dropwise with an aqueous solution of sodium nitrite $(0.5 \mathrm{gm})$. The separated product was filtered off, washed with ethanol and crystallized from acetic acid.

Yield: $59 \%$; m.p.: $208-210{ }^{\circ} \mathrm{C}$; IR (KBr, cm $\left.{ }^{-1}\right)$ : 3110-3095 (NH), $2956(\mathrm{CH}$ aromatic), $1642(\mathrm{C}=\mathrm{O}) ;{ }^{1} \mathrm{H}$ NMR $\left(\mathrm{DMSO}-d_{6}\right): \delta 5.47\left(\mathrm{~s}, 1 \mathrm{H}, \mathrm{NH}, \mathrm{D}_{2} \mathrm{O}\right.$ exchangeable), $6.41(\mathrm{~d}, 1 \mathrm{H}, J=2.6 \mathrm{~Hz}$, pyridine $\mathrm{H}-5), 6.82(\mathrm{~d}, 1 \mathrm{H}, J=2.6 \mathrm{~Hz}$, pyridine H-3), 7.15-7.63 (m, 10H, Ar-H); MS: m/z (\%) $247\left(\mathrm{M}^{+}, 35.1\right)$. Anal. Calcd. for $\mathrm{C}_{17} \mathrm{H}_{13} \mathrm{NO}$ : C, 82.57; H, 5.30; N, 5.66. Found: C, 82.32; H, 5.05; N, 5.44 .

3-Nitro-4,6-diphenylpyridin-2(1H)-one (14)

A mixture of nitric $(\mathrm{d} 1.41 ; 3 \mathrm{ml})$ and sulfuric $(\mathrm{d} 1.84 ; 3 \mathrm{ml})$ acids in glacial acetic acid $(10 \mathrm{ml})$ was gradually added to a solution of 4,6-diphenylpyridin$2(1 H)$-one $(13)(2 \mathrm{~g}, 0.008 \mathrm{~mol})$ in glacial acetic acid $(10 \mathrm{ml})$. The reaction mixture was warmed at $50^{\circ} \mathrm{C}$ for $15 \mathrm{~min}$, then poured into cold water with stirring. The separated product was filtered, washed with cold water, dried and crystallized from benzene.

Yield: $81 \%$; m.p.: 254-256 ${ }^{\circ} \mathrm{C}$; IR $\left(\mathrm{KBr}, \mathrm{cm}^{-1}\right): 3150(\mathrm{NH}), 2928(\mathrm{CH}$ aromatic), $1670(\mathrm{C}=\mathrm{O}), 1530,1335\left(\mathrm{NO}_{2}\right) ;{ }^{1} \mathrm{H}$ NMR (DMSO-d $): \delta 3.21(\mathrm{~s}, 1 \mathrm{H}$, $\mathrm{NH}, \mathrm{D}_{2} \mathrm{O}$ exchangeable), $6.56(\mathrm{~s}, 1 \mathrm{H}$, pyridine $\mathrm{H}-5), 7.43-7.81(\mathrm{~m}, 10 \mathrm{H}, \mathrm{Ar}-\mathrm{H})$; MS: $m / z(\%) 292\left(\mathrm{M}^{+}, 24.7\right)$. Anal. Calcd. for $\mathrm{C}_{17} \mathrm{H}_{12} \mathrm{~N}_{2} \mathrm{O}_{3}: 69.86 ; \mathrm{H}, 4.14 ; \mathrm{N}$, 9.58. Found: $69.64 ; \mathrm{H}, 4.32 ; \mathrm{N}, 9.81$. 
il

1

2<smiles>Cc1cc(C)c(C#N)c(=O)[nH]1</smiles><smiles>Cc1cc(C)n(N)c(=O)c1C#N</smiles>

2<smiles>Cc1cc(C)c(C#N)c(=O)[nH]1</smiles>

3<smiles>Cc1cc(C)n(N)c(=O)c1C#N</smiles>

2<smiles></smiles>

2a

Scheme 1. synthesis of compounds 2-4. 
<smiles>CC(=O)C(C=CC=CC(C)=CO)C(C)=O</smiles>

$5 \mathbf{a}$<smiles>Cc1cc(C)n(N)c(=O)c1C#N</smiles>

2

dine

$\mathrm{EtOH}$<smiles>CC(=O)/C=C(/C)Nn1c(C)cc(C)c(C#N)c1=O</smiles>

$5 b$<smiles>C=[Tl]</smiles><smiles>CC(=O)/C=N\n1c(C)cc(C)c(C#N)c1=O</smiles><smiles>C/C([O-])=C/C(C)=N/n1c(C)cc(C)c(C#N)c1=O</smiles>

5a'

$5 \mathbf{a}$

Scheme 2. Synthesis of compound 5. 

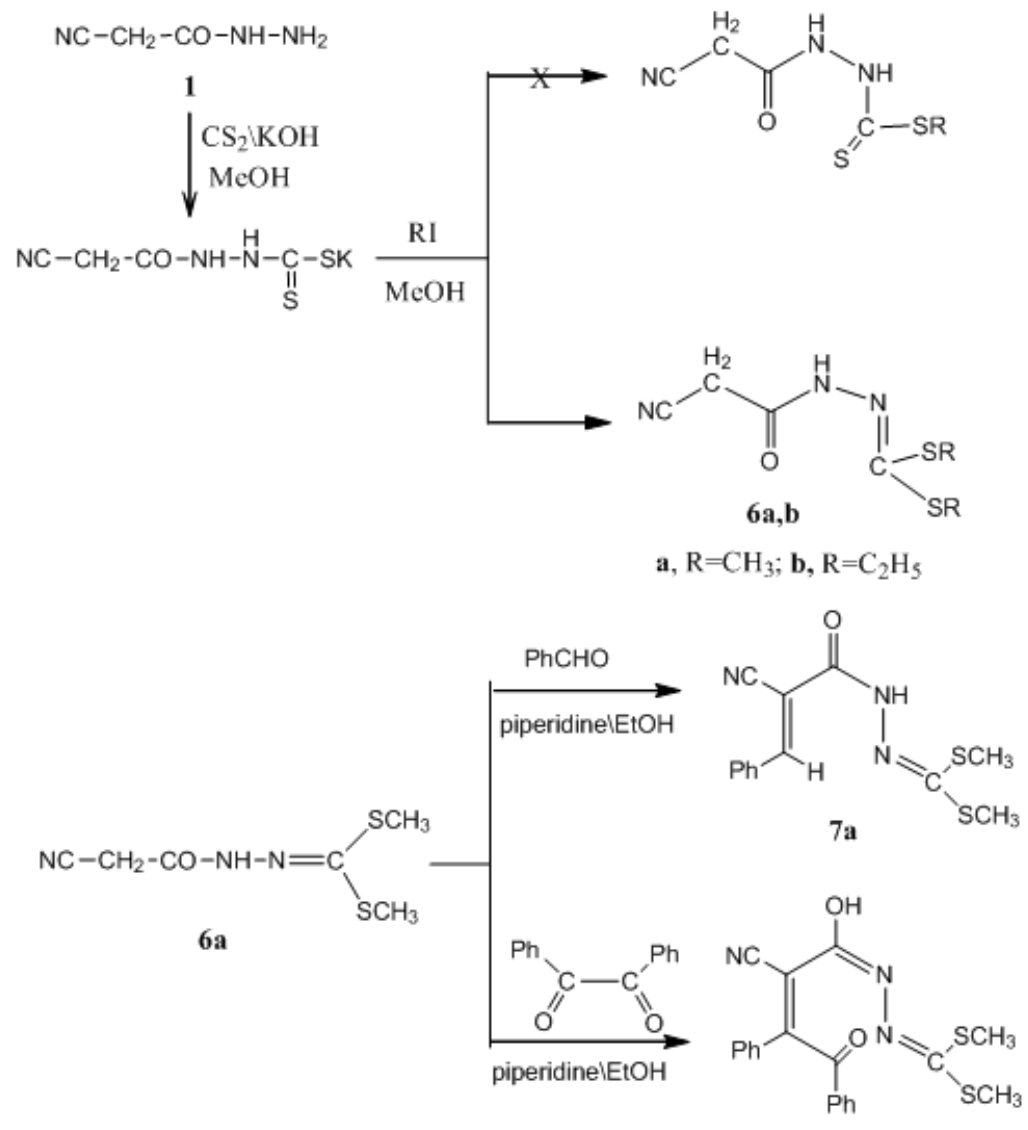

$7 \mathrm{~b}$

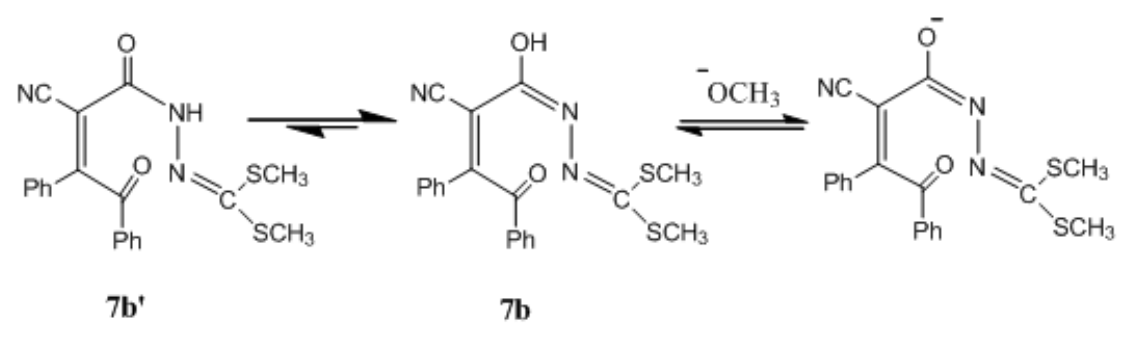

Scheme 3. Synthesis of compounds $6 a, b$ and $7 a, b$.

Egypt. J. Chem. 58, No. 6 (2015) 


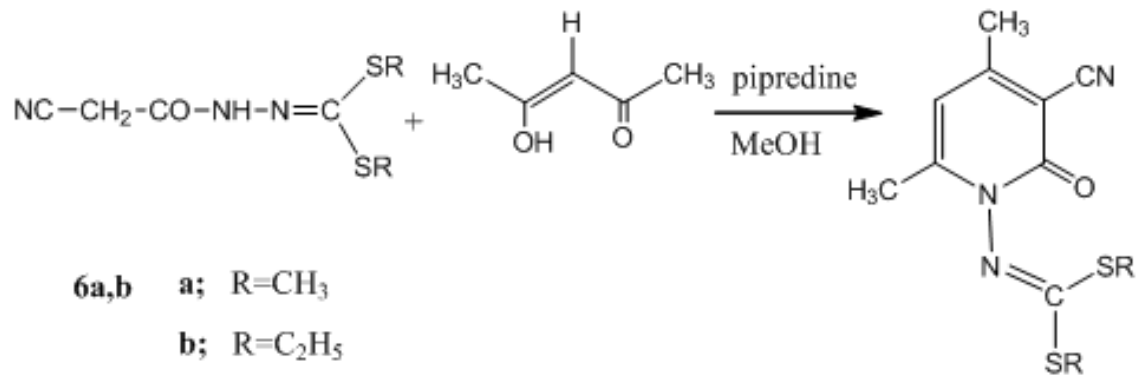

8a,b<smiles>CS/C([SiH3])=N/n1c(C)cc(C)c(C#N)c1=O</smiles><smiles>[CH]C(Cl)(Cl)Br</smiles><smiles>CS/C(S)=N/n1c(C)c([N+](=O)[O-])c(C)c(C#N)c1=O</smiles>

$8 \mathrm{a}$

Scheme 4. Synthesis of compounds 8a,b and 9.

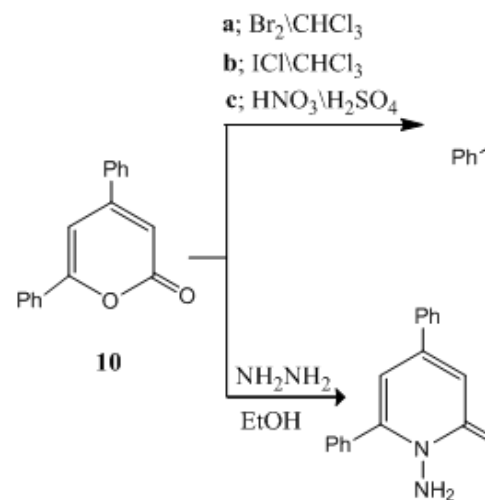

12<smiles>[R]c1c(-c2ccccc2)cc(C)oc1=O</smiles>

a; $\mathrm{R}=\mathrm{Br}$

c; $\mathrm{R}=\mathrm{NO}_{2}$

11a-c

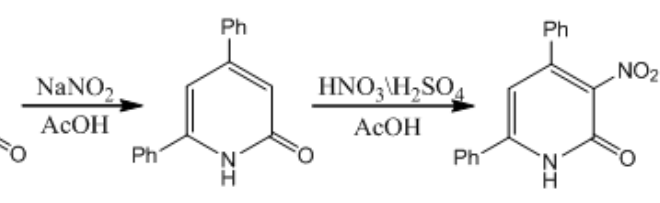

13

14

Scheme 5. Synthesis of compounds 11a-c; 12-14.

\section{Results and Discussion}

In the present investigation, some 2-pyridones containing a cyano group were synthesized. 1-Amino-3 cyano-4,6-dimethyl-2-pyridone (2) was prepared by the reaction of acetylacetone and 2-isocyanoacetohydrazide (1) in the presence of piperidine ${ }^{(21)}$. The structure of compound 2 was confirmed through 
the study of its spectral data and elemental analysis. The ${ }^{1} \mathrm{H}$ NMR spectrum showed the presence of two singlets at $\delta 2.35$ and $2.45 \mathrm{ppm}\left(2 \mathrm{CH}_{3}\right)$ beside another two singlets at $\delta 6.05$ and 6.25 for $\mathrm{NH}_{2}\left(\mathrm{D}_{2} \mathrm{O}\right.$ exchangeable $)$ and pyridine $\mathrm{H}-5$, respectively. Moreover, the electronic spectrum of compound 2 was recorded in $\mathrm{MeOH}, \mathrm{MeOH} \backslash \mathrm{H}_{2} \mathrm{SO}_{4}(0.1 \mathrm{M})$ and $\mathrm{MeOH} \backslash N a O C H_{3}(0.1 \mathrm{M})$. The spectrum in neutral and acidic solution solutions was characterized by the presence of three absorption maxima at 395, 338 and $224 \mathrm{~nm}$. However, in basic medium, the spectrum showed a new band at $206 \mathrm{~nm}$. This may be explained by assuming deprotonation of the $\mathrm{NH}_{2}$ giving the anion $2 \mathrm{a}$ (Scheme1).

4,6-Dimethyl-2-oxo-1,2-dihydropyridine-3-carbonitrile (3) was obtained by nitrous acid deamination of 1-amino-4,6-dimethyl-2-oxo-1,2-dihydropyridine-3carbonitrile (2), which was found to be completely identical to an authentic sample prepared by the condensation of acetylacetone and cyanoacetamide ${ }^{(21)}$.

Nitration of compound 2 with the conventional nitrating mixture [conc. $\mathrm{HNO}_{3} \backslash \mathrm{H}_{2} \mathrm{SO}_{4}$ (1:1)] resulted only in the isolation of the deaminated product 4,6dimethyl-2-oxo-1,2-dihydropyridine-3-carbonitrile (3). However, prolonged treatment of compound 2 with the nitrating mixture led to the formation of 3-cyano-4,6dimethyl-5-nitro-2-oxo-1,2-dihydropyridine-3-carbonitrile (4) which was also formed from the reaction of compound 3 with the nitrating mixture (Scheme 1). The presence of one singlet at $\delta 2.32 \mathrm{ppm}$ corresponding to six protons $\left(2 \mathrm{CH}_{3}\right)$ beside another singlet at $\delta \quad 10.22 \mathrm{ppm}$ for $\mathrm{NH}\left(\mathrm{D}_{2} \mathrm{O}\right.$ exchangeable) with the absence of pyridine $\mathrm{H}-5$ signal in the ${ }^{1} \mathrm{H}$ NMR spectrum, proved the formation of compound 4.

Condensation of compound 2 with acetylacetone afforded compound 5 which could be represented by any of the three possible tautomeric forms $5 \mathrm{a}, 5 \mathrm{~b}$ or $5 \mathrm{c}^{(22,23)}$ (Scheme 2). The structure of compound 5 was confirmed through the study of its spectral data and elemental analysis. Thus, the ${ }^{1} \mathrm{H}$ NMR spectrum of compound 5 showed the presence of four singlets at $\delta 1.61,1.74,2.44$ and $2.62 \mathrm{ppm}$ corresponding to the presence of four $\mathrm{CH}_{3}$ groups. Also, the presence of signal at $\delta 5.35 \mathrm{ppm}$ for $\mathrm{C}-\mathrm{H}$ proton of the acetylacetone moiety and $11.54\left(\mathrm{OH}, \mathrm{D}_{2} \mathrm{O}\right.$ exchangeable) ppm supported that the condensation product 5 exist in the enolimine structure $5 \mathrm{a}^{(23)}$. Moreover, the electronic spectrum of compound 5 was recorded in $\mathrm{MeOH}, \mathrm{MeOH} \backslash \mathrm{H}_{2} \mathrm{SO}_{4}(0.1 \mathrm{M})$ and $\mathrm{MeOH} \backslash N a O C H_{3}(0.1 \mathrm{M})$ (Fig. 1) (see the experimental section) showed the presence of two isobestic points at 272 and $238 \mathrm{~nm}$., a fact that denotes the presence of an acid-base equilibrium. A comparison of the spectra revealed that the band at $284 \mathrm{~nm}$ in the neutral species becomes more intense in basic medium and diminishes in acid solution which confirmed that compound 5 exists in the enol-imine structure 5a. The latter compound exists in equilibrium in the neutral solution. In acidic solution the form 5 a predominates, while the anion 5a' is the predominant form in the basic medium (Scheme 2).

Egypt. J. Chem. 58, No. 6 (2015) 


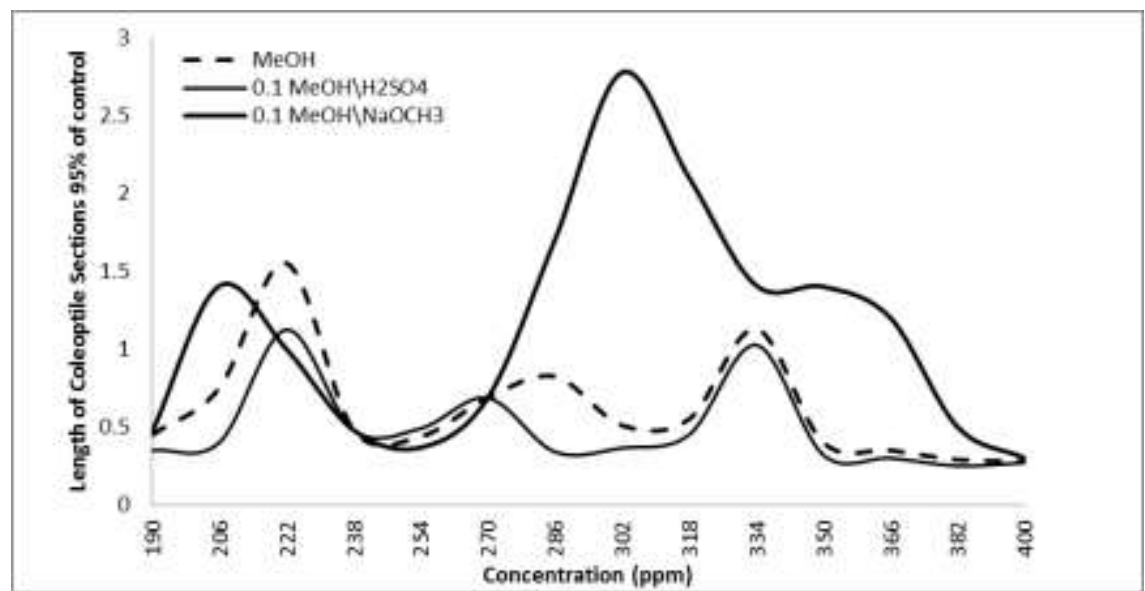

Fig. 1. Electronic spectrum of compound 5.

In the present investigation compound 2 failed to react with carbon disulfide and methyl iodide ${ }^{(24,25)}$. Therefore, a new synthetic strategy had to be adopted by the introduction of the sulfur moiety to the cyanoacetohydrazide 1 before condensation with acetylacetone. However, when cyanoacetohydrazide 1 was subjected to the standard carbodithioation experimental condition ${ }^{(24,25)}$, the only isolated product was shown to be dialkyl (2-isocyanoacetyl) carbonohydrazonodithioate $\left(6 \mathrm{a}, \mathrm{b} ; \mathrm{R}=\mathrm{CH}_{3}, \mathrm{C}_{2} \mathrm{H}_{5}\right)$ by using 2 moles of each of $\mathrm{KOH}$ and alkyl iodide. The novel products $6 \mathrm{a}, \mathrm{b}\left(\mathrm{R}=\mathrm{CH}_{3}, \mathrm{C}_{2} \mathrm{H}_{5}\right)$ were characterized through a study of their spectral data, together with some condensation reactions with simple carbonyl and $\alpha$-dicarbonyl compounds.(Scheme 3). Thus, the ${ }^{1} \mathrm{H}$ NMR spectrum of compound $6 \mathrm{~b}$ (as an example) showed the presence of triplet and quartet at $\delta 1.35$ and $3.15 \mathrm{ppm}$ corresponding to the $2 \mathrm{CH}_{3}$ and $2 \mathrm{CH}_{2}$ protons of the two ethyl groups. The two protons of the active methylene group being magnetically nonequivalent showing double doublet at $\delta 3.91 \mathrm{ppm}$ beside a broad singlet at $\delta 10.55 \mathrm{ppm}$ for $\mathrm{NH}$ group $\left(\mathrm{D}_{2} \mathrm{O}\right.$ exchangeable). Moreover, ${ }^{13} \mathrm{C}$ NMR spectrum showed $\delta: 13.8$, $15.1\left(2 \mathrm{CH}_{3}\right), 24.4,26.1,26.7\left(3 \mathrm{CH}_{2}\right), 116.2(\mathrm{CN}), 158.1(\mathrm{C}=\mathrm{N}), 165.4(\mathrm{CO})$.

Condensation of $6 \mathrm{a}$ with either benzaldhyde or benzil afforded the condensation products $7 \mathrm{a}, \mathrm{b}$. The ${ }^{1} \mathrm{H}$ NMR spectrum of $7 \mathrm{~b}$ (as an example) showed the presence of two methyl signals at $\delta 2.11$ and $2.42 \mathrm{ppm}$, together with multiplet at $\delta 7.21-7.64 \mathrm{ppm}$ corresponding to the ten aromatic protons. The singlet at $\delta 7.90 \mathrm{ppm}$ may be attributed to the $\mathrm{OH}$ proton of the enol-imine form $7 \mathrm{~b}\left(\mathrm{R}=\mathrm{CH}_{3}\right)$, a behavior often characterizing such systems ${ }^{(23)}$. At the time being, ${ }^{13} \mathrm{C}$ NMR spectrum revealed $\delta$ : 19.8, $20.1\left(2 \mathrm{CH}_{3}\right), 119.3(\mathrm{CN}), 162.9(\mathrm{C}=\mathrm{N})$, $173.1,179.1$ (2CO). A further elucidation of the structure of compound $7 \mathrm{~b}$ was achieved through a study of its electronic spectrum in $\mathrm{MeOH}, \mathrm{MeOH} \mathrm{H}_{2} \mathrm{SO}_{4}(0.1$ M) and $\mathrm{MeOH} \backslash \mathrm{NaOCH}_{3}(0.1 \mathrm{M})$ (see the experimental section). The UV spectrum is characterized by the appearance of four absorption maxima at 580, 387,245 and $282 \mathrm{~nm}$ which were not affected neither in acidic nor in basic 
media. This phenomenon could only be explained by assuming the predominance of the enol-imine form (7b) rather than the keto-imine (7b') in the neutral solution (Scheme 3)

2 - Oxopyridin -1 (2H) -yl) carbonimidodithioate derivatives 8a,b were synthesized from the reaction of $6 a, b$ with acetylacetone in the presence of piperidine as a base. The ${ }^{1} \mathrm{H}$ NMR spectrum for $8 \mathrm{a}$ (as an example) exhibited the presence of four methyl groups' singlets at $\delta 2.20,2.35,2.55$ and $2.65 \mathrm{ppm}$ beside another singlet at $\delta 6.32 \mathrm{ppm}$ corresponding to pyridine $\mathrm{H}-5$. In addition, the ${ }^{13} \mathrm{C}$ NMR spectrum showed $\delta 13.9,15.1,18.5,20.4\left(4 \mathrm{CH}_{3}\right), 116.4(\mathrm{CN}), 157.1(\mathrm{C}=\mathrm{N})$, $180.1(\mathrm{CO})$.

It was reported that the $\mathrm{S}-\mathrm{CH}_{3}$ group could be substituted with halogen ${ }^{(26)}$. However, treatment of compound $8 \mathrm{a}$ with $\mathrm{Br}_{2} \mathrm{CCCl}_{4}$ led to the introduction of bromine at the five position of the 2-pyridone ring giving compound 9 . The ${ }^{1} \mathrm{H}$ NMR spectrum of compound 9 was almost identical with that of $8 \mathrm{a}$, except for the disappearance of the C-5 proton signal (Scheme 4).

A well-established route for the synthesis of 2-pyridones involves the reaction of $2 \mathrm{H}$-pyran-2-ones with ammonia, amines as well as hydrazines ${ }^{(20)}$ Therefore, in the present work a series of 3-substituted 4,6-diphenyl-2H-pyran-2one (11a-c) were prepared from 4,6-diphenyl-2H-pyran-2-one (10). The structure of $11 \mathrm{a}-\mathrm{c}$ was in agreement with their spectral data and elemental analyses (see experimental section). 3-Substituted 4,6-diphenyl-2H-pyran-2-one (11a-c) failed to react with hydrazine hydrate and only resinous product was formed which could not be identified. Only, 4,6-diphenyl-2H-pyran-2-one (10) reacted with hydrazine hydrate and afforded 1-amino-4,6-diphenylpyridin-2(1H)-one (12). The structure of the latter compound was confirmed on the basis of its spectral data and elemental analysis. The ${ }^{1} \mathrm{H}$ NMR spectrum of compound 12 exhibited two doublets $(J=2.6 \mathrm{~Hz})$ at $\delta 6.45$ and $6.86 \mathrm{ppm}$ due to long rang coupling between $\mathrm{C}-5$ and $\mathrm{C}-3$ protons, respectively. Also, the presence of broad singlet at $\delta 5.07 \mathrm{ppm}\left(\mathrm{D}_{2} \mathrm{O}\right.$ exchangeable) for $\mathrm{NH}_{2}$ protons beside multiplet corresponding to the ten aromatic protons at $\delta 7.23-7.74 \mathrm{ppm}$. Deamination of compound 12 with sodium nitrite in glacial acetic acid afforded 4,6-Diphenylpyridin-2(1H)one $(13)^{(27)}$. Finally, 3-nitro-4,6-diphenylpyridin-2(1H)-one (14) was prepared from the nitration of compound 13 using the conventional nitrating mixture [conc. $\left.\mathrm{HNO}_{3} \backslash \mathrm{H}_{2} \mathrm{SO}_{4}(1: 1)\right]$. The structure of the latter compound was confirmed through the study of its spectral data and elemental analysis (Scheme 5).

\section{References}

1. Kuethe, J.T., Wong, A. and Davies, I.W., Synthesis of disubstituted imidazo[4,5b]pyridin-2-ones. J. Org. Chem. 69 (22), 7752-7754 (2004).

2. a; Bailey T., Goe, G. and Scriven, E., In Heterocyclic Compounds; Newkome, G. R., (Ed.); Wiley: New York, 14 (5), 1-4 (1984). b; Smith D., In: Comprehensive Organic Chemistry; Sammes, P.G., (Ed.) Pergamon: Oxford, 4, 3-5 (1979).

Egypt. J. Chem. 58, No. 6 (2015) 
3. Kibou, Z., Cheikh, N., Villemin, D., Choukchou-Braham, N., Mostefa-Kara, B. and Benabdallah M., A simple and efficient procedure for 2-pyridones synthesis under solvent-free conditions. Int. J. Org. Chem. 1, 242-249 (2011).

4. Cherry, K., Abarbri, M., Parrain, J-L. and Duche^ne, A., Regio- and selective synthesis of 4,6-disubstituted-2-pyridones.Tetrahedron Lett. 44, 5791-5796 (2003).

5. Elassar A., Synthesis and reactions of 3-cyano-4,6-dimethyl-2-pyridone. J. Heterocyclic Chem. 48, 272-278 (2011).

6. Cocco, M.T., Congiu, C. and Onnis, V., Synthesis and antitumour activity of 4hydroxy-2-pyridone derivatives. Eur. J. Med. Chem. 35 (5), 545-552 (2000).

7. Curran, D.P. and Liu, H., New $4+1$ radical annulations. A formal total synthesis of ( \pm )-camptothecin. J. Am. Chem. Soc. 114, 5863 (1992)

8. Cox, R. J. and O'Hagan, D., Synthesis of isotopically labelled 3-amino-2-phenylpropionic acid and its role as a precursor in the biosynthesis of tenellin and tropic acid. J. Chem. Soc. Perkin Trans. 1, 2537-2540 (1991).

9. Manna, F., Chimenti, F., Bolasco, A., Rossi, F., Cenicola, M.L., D'Amico, M. and Parrillo, C., Antiinflammatory, analgesic and antipyretic n-acetyl $\Delta^{2} 2$-pyrazolines and dihydrothienocoumarines. Ph. Res. 26, 267 (1992).

10. Williams D.R., Lowder, P.D. and Gu Y-G., Studies toward funiculosin. Intramolecular carbonyl condensations using carboxamidimidazolide intermediates. Tetrahedron Lett. 38 (3), 327-330 (1997).

11. Kozikowski, A.P., Campiani, G., Sun, L-Q., Wang, S., Saxena, A. and Doctor, B.P., Identification of a more potent analogue of the naturally occurring alkaloid huperzine A. predictive molecular modeling of its interaction with AChE. J. Am. Chem. Soc. 118, 11357-11362 (1996).

12. Dragovich, P.S., Prins, T.J., Zhou, R., Brown, E.L., Maldonado, F.C., Fuhrman, S.A., Zalman, L.S., Tuntland, T., Lee, C.A. and Worland, S.T., Structure-based design, synthesis, and biological evaluation of irreversible human rhinovirus 3C protease inhibitors. 6 . Structure-activity studies of orally bioavailable, 2-pyridonecontaining peptidomimetics. J. Med. Chem. 45 (8), 1607-1623 (2002).

13.Murray, T.J. and Zimmerman, S.C., 7-Amido-1,8-naphthyridines as hydrogen bonding units for the complexation of guanine derivatives: The role of 2-alkoxyl groups in decreasing binding affinity. Tetrahedron Lett. 36, 7627-7630 (1995).

14.Jones, G., In Comprehensive Heterocyclic Chemistry II; Oxford: Pergamon Press; Katritzky, A., Rees, C. W., Scriven, E. F.,( Eds.), pp. 395-510 (1996).

15. Abu-Shanab, F.A., Redhouse, A.D., Thompson, J.R. and Wakefield, B.J., Synthesis of 2,3,5,6-tetrasubstituted pyridines from enamines derived from N,N-dimethylformamide dimethyl acetal. Synthesis, 5, 557-560 (1995). 
16. Jones, W.D., Schnettler, R.A. and Huber E.W., A convenient synthesis of 5-acyl-6substituted 3-cyano-2(1H)-pyridinones. J. Heterocyclic Chem. 27 (3), 511-518 (1990).

17. Fukatsu, H., Kato, Y., Murase, S. and Nakagawa, S., Synthesis and cardiotonic activity of 5-(2-substituted thiazol-4-yl)-2-pyridones and thiazolo[4,5-f] quinolinones. Heterocycles, 29 (8), 1517-1528 (1989).

18. Sircar, I., Duell, B.L., Bristol, J.A., Weishaar, R.E. and Evans D.B., Cardiotonic agents. 5. 1, 2- dihydro - 5 - [ 4 - ( $1 H$-imidazol - 1 - yl ) phenyl ] - 6-methyl - 2 oxo -3 - pyridinecarbonitriles and related compounds. Synthesis and inotropic activity. J. Med. Chem. 30 (6), 1023-1029 (1987).

19. Abadi, A.H., Ibrahim, T.M., Abouzid, K.M., Lehmann, J., Tinsley, H.N., Gary, B.D. and Piazza, G.A., Design, synthesis and biological evaluation of novel pyridine derivatives as anticancer agents and phosphodiesterase 3 inhibitors. Bioorg. Med. Chem. 17 (16), 5974-5982 (2009).

20. EL-Kholy, I.E., Rafla, F.K. and Mishrikey, M.M., Pyrone series. Part XI. Reactivity of 4,6-diaryl-2-pyrones and cyclopropane derivatives obtained from 2pyrazolines. J. Chem. Soc. (C), 1578-1584 (1970).

21. Ried, W. and Meyer, A., Über die Verwendung von Cyanacethydrazid zur Darstellung von Stickstoffheterocyclen. I. Eine einfache Synthese von N-amino- $\alpha$ pyridonen. Chem. Ber. 90, 2841-2848 (1957).

22. Fabian, J., Legrand, M. and Poirer, P., Infrared spectroscopy of carbohydrates: A review of the literature. Bull. Soc. Chim., France, 1499 (1956).

23. Rateb, L., Azmy, B., Nashed, M.A. and Iskander, M.F., Hydrazine derivatives of ßdicarbonyl compounds, part I Studies on some benzoylacetaldehyde aroylhydrazones. Z. Natruforsh, 83b, 1527-1534 (1978).

24. Merchan, F., Garin, J. and Melendez, E., $8^{\text {th }}$,International Congress of Heterocyclic chemistry, Austria, p. 152 (1981).

25. Kamel, M.M. and Megally, Abdo N.Y., Synthesis of novel 1,2,4-triazoles, triazolothiadiazines and triazolothiadiazoles as potential anticancer agents. Eur. J. Med. Chem. 86, 75-80 (2014).

26. Garin, J., Melendez, E., Merchan, F.L. and Tejero, III. International Congress of Therapeutic Chemistry (Espain) Abs. Book C-20 (1983).

27. Basu, U., Synthesis and reaction of 4,6-diphenyl-2H-pyran-2-one. J. Indian Chem. Soc.7, 481, 851 (1930). 


\section{استخدام الأسيتيل اسيتون فى تحضير مشتقات بيريدين 2-اون} لطيف راتب ، مرقس ميلاد مشرقى ، جورجى عطالله مينا" ، باسم عزمى سلوانس ،

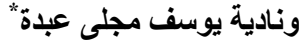
قسم الكيمياء ـ كلية العلوم و *قسم الكيمياءـ كلية التربيةـ جامعة الأسكندريةـ الاسكندرية ـ

فى هذا البحث نم تحضير سلسلة من مشتقات بيريدين 2-اون من خلال تفاعل

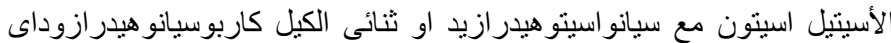

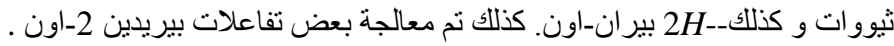

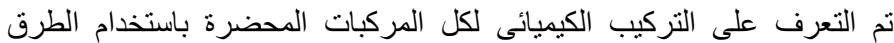

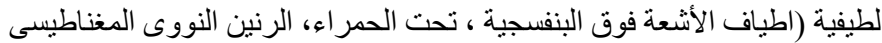
الكرن، الو ${ }^{1}$ H NMR الكربون، الهيدروجين، النيتروجين و الكبريت. 